\title{
Transformative art: art as means for long-term neurocognitive change
}

\author{
Son Preminger * \\ School of Psychology, Interdisciplinary Center Herzliya, Herzliya, Israel
}

\section{Edited by:}

Idan Segev, The Hebrew University of Jerusalem, Israel

Reviewed by:

Idan Segev, The Hebrew University of Jerusalem, Israel

Lutz Jäncke, University of Zurich,

Switzerland

*Correspondence:

Son Preminger, School of

Psychology, Interdisciplinary Center Herzliya (IDC), Herzliya 46150, Israel. e-mail: sonpreminger@gmail.com
Every artwork leads to a unique experience by the observer or participant, may it be sensory, emotional, cognitive, interactive, or spiritual experience. At the neurobiological level, such experiences are manifested as activation of the corresponding neural networks. Neuroscience has demonstrated that experience, in particular repeated experience, can cause a long-term change in the involved brain circuits (experience-dependent plasticity). This review will discuss the molding and transformative aspect of arts, examining how repeated and on-going experience of arts may alter cognitive, emotional, and behavioral patterns as well as their underlying neural circuits. The application of this approach to cognitive training and neuropsychological rehabilitation methods will be addressed as well. In addition, it will be suggested that this approach to art, as a long-term transformative medium, may lead to a novel viewpoint on art and a different approach to its creation. Artists can design artworks that aspire to form, in addition to one-shot influencing experience, on-going experiences which gradually create a lasting change, possibly improving audiences' neuropsychological functions.

Keywords: plasticity, neurocognitive, art, training, perceptual learning, rehabilitation, video games, improvisation

\section{ART AS A NEUROCOGNITIVE EXPERIENCE}

Art is a medium of inducing experiences. Artistic experiences can be a vehicle to convey meanings, a way to provide pleasure, or means for self-expression and communication. Every artwork leads to a mental experience by the observer, participant, or experiencer. Experiencing an art work commonly involves perceptual processes-for example, plastic arts engage low-level visual processes such as orientation and edge detection, as well as higher-level processes, such as object recognition and its segregation from background. Similarly to daily life experiences, an artistic experience would involve additional cognitive processes such as executive functions, memory, emotion, and other high-level cognitive processes. The engagement of executive functions such as working memory and attention, are at the grounds of many artistic experiences as being able to assemble the different pieces of the presented work and being able to avoid distractions to be captivated by the artwork, are an essential for experiencing it (Dudai, 2008). Intrinsic processes such as autobiographic memory, emotion, and theory of mind may be driven by perceptual elements and provide meaning and essence to the artwork.

The specific combination of cognitive functions engaged by an art piece depends on the art form, the particular piece and the observer's unique experience. For example, classical art forms such as plastic arts, music, and film, relay mainly on perception to drive an artistic mental experience. On the other hand, interactive arts such as interactive installations or video games involve also motor functions and behavioral control as part of the induced experience. Importantly, people performing or practicing arts such as musicians or actors, also engage such action-related mechanisms while they practice their arts.

\section{THE EFFECT OF EXPERIENCE ON THE BRAIN EXPERIENCE AS NEURAL NETWORK ACTIVITY}

At the neurobiological level, mental experiences are manifested as activation of the corresponding neural networks at the visual and auditory cortices, attention and memory networks, emotional brain regions, frontal and other brain regions, and their combinations. The distribution of the neuronal activity patterns depends on the particular cognitive processes that form and drive the experience (Kandel et al., 2000). Whether experience can be captured merely by biological correlates, or there are components which can not be described in physical terms and are only introspectively accessible (e.g., the sensation of seeing red; termed Qualia; Koch, 2004), has been intensively debated in philosophy. Here I will focus on the aspects of experience that are manifested and can be measured on a neurobiological or behavioral level.

\section{EXPERIENCE-INDUCED BRAIN PLASTICITY}

A key characteristic of the brain is its capacity to change as a result of experience (see reviews in Buonomano and Merzenich, 1998; Kolb and Whishaw, 1998; Kandel, 2001; Pascual-Leone et al., 2005). Neural learning theories are commonly based on the notion termed experience-dependent plasticity which states that activation of a neural network leads to a modification of the corresponding synaptic connections (Hebb, 1949). Multiple human and animal neuroscience studies have demonstrated that experiences can lead to brain changes ranging from cellular modifications to formation of new synaptic connections and reorganization of cortical networks (reviews in Buonomano and Merzenich, 1998; Kolb and Whishaw, 1998; Kandel, 2001; Kelly and Garavan, 2005; Pascual-Leone et al., 
2005). In particular, studies suggest experience-based neural plasticity processes underlie the change and adaptation of visual perception capabilities (Kourtzi and DiCarlo, 2006; Sagi, 2010). Various experimental procedures were designed to induce longterm changes of perception, for example improvement of lowlevel perceptual abilities (e.g., Karni and Sagi, 1991; Recanzone et al., 1993; reviews Buonomano and Merzenich, 1998; Sagi, 2010), as well as higher perceptual abilities such as learning to recognize objects (Kourtzi and DiCarlo, 2006), and gradually transforming perceptual object recognition (Preminger et al., 2007, 2009b). Similar experience-dependent plasticity characteristics have been demonstrated in the motor domain (e.g., Newell and Rosenbloom, 1981; Karni et al., 1998; Bezzola et al., 2011) as well as higher-order cognitive functions such as working memory (Mahncke et al., 2006; Chein and Morrison, 2010). Many studies have also demonstrated that behavioral changes that are induced by repetitive training are accompanied by long-term changes in brain activity and structure (e.g., Karni et al., 1998; Li et al., 2009; Bezzola et al., 2011; review in Kelly and Garavan, 2005). The experience-based plasticity approach has been also applied successfully to induce neural changes and functional improvement in cognitive functions for people with various cognitive impairments. For example, training has been shown to improve visual perception in amblyopia (Polat et al., 2004), and motor function in stroke (Hlustík and Mayer, 2006).

Importantly, studies of perceptual and motor experiencedbased plasticity have shown specific conditions and constraints for perceptual and motor learning. These principles could serve as guidelines and stating point when considering more broad plasticity principles. Major factors include: time-scales of learning, specificity vs. difficulty, interference, order of learning, training intensity and duration, context, motivation, and arousal, feedback, and variability. A full description of these principles is out of the scope of this review, the details can be found in (Green and Bavelier, 2008; Sagi, 2010; Preminger, 2011). In addition, other insights from learning research may be applied. One important example is the role of deliberate practice in developing expertise-it has been suggested that the amount of deliberate practice is a major factor influencing the level of expertise in various domains (Ericsson et al., 1993).

It is important to note that the focus of this review is on mechanisms of change of cognitive function which are based on repeated or ongoing experiences which gradually modify synaptic connections and consequently cognitive processing. This is a major mechanism by which many of our cognitive functions adjust and improve over time. Other mechanisms for inducing long-term changes exist, such as one-shot learning, which is often seen in episodic memory (Roediger et al., 2007), where one-time experiences may be remembered, and may induce long-term neural changes (Ludmer et al., 2011). When considering a lasting effect of their work most artists probably have in mind such long-term effect due to single impression. Here I put forward a different approach, proposing that art could be viewed as a medium that by instigating repeated experiences may induce long-term changes and serve as means for modification, improvement, and rehabilitation of various cognitive functions.

\section{THE LONG-TERM EFFECT OF ARTISTIC EXPERIENCE ON THE BRAIN}

There are many examples of potentially transformative aspects of experiencing art, such ongoing exposure to plastic art and music influencing our perception and sense of beauty, or watching films or television affecting our social and behavioral patterns. Although multiple studies explore brain activation while experiencing various art mediums: plastic arts (Zeki, 1999; Ishai, 2011), music (Stewart, 2008), and films (Hasson et al., 2008, 2009), research of the long-term effect of repeated and ongoing exposure to artistic experiences is limited. Only a few studies examine the lasting effect of perceiving art on the brain, as it is not easy to isolate the effect of such ongoing experiences and to follow them long-term. One recent example is a functional magnetic resonance imaging ( $\mathrm{fMRI}$ ) study conducted on a short training in recognizing objects in cubist paintings (Wiesmann and Ishai, 2010), which has demonstrated that trained subjects where better than non-trained ones at the trained task and showed enhanced brain activity in the parahippocampal cortex.

Studies of the long-term effects of art often focus on the effects of practicing art-some by comparing artists to nonartists, and others by examining effects on purposely trained subjects. Specifically, the effect of practicing music on brain and cognition has been studied considerably, demonstrating structural and functional specializations (Stewart, 2008; Jancke, 2009). For example, a brain imaging study comparing brains of musicians and non-musicians demonstrated that musicians have more gray matter in primary auditory cortex (Schneider et al., 2005). Another study, which compared electroencephalography (EEG) event-related potentials during presentation of auditory stimuli between children with musical training and ones without, has demonstrated that musical training enhances the sensitivity of the auditory system (Meyer et al., 2011).

The practice of visual arts has also been examined. For example, a recent EEG study (Kottlow et al., 2011) found decreased upper alpha waves in artists vs. non-artists during various drawing tasks, suggesting that cognitive functioning, semantic memory, and object recognition are enhanced in artists. Another study, comparing eye-movements of visual artists to non-artists, showed that while viewing pictures artists spend more time scanning structural and abstract features, whereas non-artists focus more on human features and objects (Vogt and Magnussen, 2007).

Importantly, some of these studies point to the fact that practicing art may influence not only perception but also higher-order cognitive processes such as attention, memory, and executive functions. Of particular potential for such effects on high-level cognitive functions are also art domains which are centered on action by the artist, such as performing arts, or by the experiencer, such as interactive arts (e.g., interactive installations and theater, video games). In the following, I elaborate on the long-term effects of two such examples_-video games and improvisation in theater and music.

\section{VIDEO GAMES}

Video games are one domain in which the long-term effect on brain and cognition was studied more extensively. This is 
probably due to the fact that video games are designed for repetitive experience and are used as such. Although the inclusion of video games as part of arts is debated (Jenkins, 2000; Kroll, 2000), contemporary approaches appreciate the artistic quality of video games and their strong influence on modern culture (Smuts, 2005). In the context of our discussion, since video games clearly induce experience, are rewarding and engaging, and often communicate meaning, we view them as artistic experience. Studies of the long-term effects of playing video games have demonstrated that action games improve performance in many sensory, perceptual, and attentional tasks that extend well beyond the trained conditions (Green and Bavelier, 2006a). Playing action video games was shown to improve visual reaction times (review in Dye et al., 2009), enhance visuomotor coordination (e.g., Drew and Waters, 1986), improve spatial visualization skills (e.g., Dorval and Pepin, 1986), enhance various aspects of attention, (Green and Bavelier, 2003, 2006b; Feng et al., 2007), and improve probabilistic inference (Green et al., 2010). These effects were demonstrated by comparing gamers to non-games or by studying training effects on a purposely trained group vs. a control group. Recently it was demonstrated that at least some cognitive differences between gamers and non-gamers result either from pre-existing group differences or from very extensive game experience (Boot et al., 2008), thus studying gamers to infer the neurocognitive effects of games should be done with caution.

Altogether, video game playing has been shown to result in a broad spectrum of generalized performance enhancements in perceptual abilities as well as high-level cognitive functions. It has been suggested that probabilistic inference may be a general learning mechanism underlying these wide-range improvements (Green et al., 2010). However, these cognitive enhancements could also be attributed to several other factors. First, video games involve a more holistic and variable experience than classical perceptual learning paradigms (Green and Bavelier, 2008; Sagi, 2010) and thus are more ecological. Second, experiencing video games involves behaving and acting in an intensive and dynamic environment which may lead to enhanced learning (Achtman et al., 2008). Finally, rewards in video games are very dominant and timely assigned, which could be another reason for improved learning (Achtman et al., 2008). These characteristics of video games are also in contrast to art domains where experience involves mainly perception, which incites thoughts and emotions but does not involve action, and often is less intense. The value of these various characteristics and the principles underlying the generalization power of different training and practice formats will be further discussed later.

\section{IMPROVISATION IN THEATER AND MUSIC}

To improvise means to grasp the present circumstances and act accordingly and appropriately, not being constrained by habits, fears, or conformity, nor behaving randomly or by impulses (Nachmanovitch, 1990). Performance of such "non-default" behavior in complex and novel contexts characterizes the functions of the frontal lobes (Mesulam, 2002). Although we all improvise in our daily life when we act in complex or novel situations, improvisation is also strongly associated with improvised performance in the arts, specifically in music and theater.
Recently several neuroimaging studies have examined brain activation during musical improvisation and demonstrated activation of prefrontal regions (Bengtsson et al., 2007; Limb and Braun, 2008; Berkowitz and Ansari, 2008). Furthermore, it has been noted that many of the exercises and practices performed by improvisation actors share high resemblance to classical neuropsychological assessments for prefrontal functions (Preminger, 2009a, 2011). Consequently it was suggested that improvisation training can serve as training and rehabilitation for prefrontal functions (Preminger, 2009a, 2011), proposing that a major benefit of such training is its resemblance to real-life, leading to a higher potential for transfer. Brain activity during some specific improvisation tasks and the long-term effect of their continued practice has been examined and demonstrated using fMRI and computerized paradigms that simulate improvisation exercises to allow for scientific investigation (Preminger et al., 2008, 2010a; Loya et al., 2010).

So far I focused on describing how a given art experience affects the brain. Given that brain and cognition have the capacity to be molded by artistic experiences, art can be created in a way that takes this knowledge into account and utilizes it to generate transformative experiences with particular artistic or rehabilitational goals in mind.

\section{ART AS ENGINEERED EXPERIENCE}

Although the nature of aesthetic experience has been forcefully debated for centuries, there is a general agreement in the western culture about the term aesthetic experience, and various suggestion for its components where proposed. For example, Ramachandran and Hirstein proposed the Eight laws of artistic experience-common principles for how visual artistic experiences excite human cognition and brain (e.g., grouping, symmetry, and peak shift principles; Ramachandran and Hirstein, 1999). Furthermore, many art theorists agree that overall there are many commonalities in the way different people (from same culture) experience a given artwork. Some even claim that the ability to convey a consistent experience is a criterion for the quality of the artwork. Indeed, previous studies have demonstrated that eye-movement of different viewers while viewing the same famous paintings share a lot in common (Yarbuz, 1976). Similar results where recently obtained with viewers of commercial movies (Hasson et al., 2004, 2008). Furthermore, neuroscientists have suggested that at least at an elementary level, what happens in different brains when viewing works of art is very similar (Zeki, 1998). Neuroimaging studies have demonstrated a common brain activity pattern when subjects viewed paintings (Ishai, 2011), or when subjects listened to musical pieces (Stewart, 2008). Furthermore, it was demonstrated that when viewing a movie the time course of brain activity in sensory and association cortices correlated across viewers (Hasson et al., 2004). Nevertheless, correlation across viewers was not seen in all regions, in particular not in frontal cortical regions. The subjective aspect of perception was also demonstrated by a recent study of conscious perception using Dali's ambiguous painting (Smith et al., 2006) which demonstrated that different image components drive different observers to reach the same perceptual awareness. 
Clearly, the ability to control the cognitive processes depends on the art medium (e.g., plastic art vs. cinema vs. music). Furthermore, the inclination of artists to induce a common experience across all viewers varies. One clear example is the different approaches taken by various film directors and theorists: whereas the Russian montage editing approach promoted "engineering" new movie experiences by combining different perceptual components together, the other side of the spectrum can be represented by André Bazin who advocated films which are "objective reality," leaving the viewer free to have his own subjective experience. Along these lines, it has been recently demonstrated that different films by different directors vary in the consistency of brain activation across viewers, where structured movies such as Bang! You're Dead by Hitchcock, elicited a wide range of brain regions where activity was correlated across subjects who viewed the movie (Hasson et al., 2008). On the other hand, an un-structured movie of a daily-life scene very few regions demonstrated correlation across subjects (Hasson et al., 2008).

In summary, at least to some extent, artists can be viewed as experts in controlling and manipulating humans' perceptions as well as the emotional and cognitive experience that they induce.

\section{ENGINEERING ARTISTIC EXPERIENCES FOR LONG-TERM CHANGE}

This capacity of the artist to engineer experiences, and thus brain activity, can be, and has been, taken one step further. Art can be used to induce long-term changes in cognition, emotion, and behavior. One example for a similar approach was applied in the past, again by Soviet film directors (Dziga Vertov, Sergei Eisenstein), who promoted the usage of cinema as a propaganda tool for emotional and psychological influence on their audience for ideological purposes. Likewise, advertizing and marketing experts have been using similar principles over and over to achieve their commercial goals by changing behavioral, emotional and thought patterns (termed NeuroMarketing; e.g., Rothschild et al., 1986; Rothschild and Hyun, 1990; McClure et al., 2004).

These approaches are aligned with the theory of experiencebased plasticity by their similar reliance on repeated experience for modifying cognition. However, in contrast to these approaches who put in front of their eyes a goal which is external to the goals of their experiencer (e.g., buy some product, support a political movement), the approach presented here places focus on the consideration of art as a method for influencing and optimization of cognitive and brain functions to the benefit and value of the observer or experiencer, similarly to the approach taken by rehabilitation disciplines in designing cognitive training programs.

\section{THE VALUE OF ART AS A TRANSFORMATIVE EXPERIENCE}

To understand how artistic experiences may contribute in designing transformative experiences, the first question that comes to mind is what is unique about artistic experience as opposed to other experiences such as daily-life experience or classical cognitive training experiences. The uniqueness of artistic and aesthetic experience is a broad and complex topic which has been addressed extensively by artists and philosophers. I will focus here mainly on the aspects that might be relevant to the transformative aspect of art. What qualities of artistic experience may cause it to be a better transformative experience than a daily life experience? What value can if offer over classical cognitive training paradigms?

Some theorists describe aesthetic experience with emphasis on unity, holism, and emotional experience. "In aesthetic experience ... there is completeness and unity and necessarily emotion." (Dewey, 1934). Likewise it was suggested (Beardsley, 1958) that all aesthetic experiences have in common the following features: "focus, intensity, and unity, where unity is a matter of coherence and of completeness." This view, which stresses the immersive and holistic nature of the experience of art, points to the advantage of art as a training tool due to its captivating and engaging nature which presumably leads to focus, attention, and motivation which are know to enhance learning (Green and Bavelier, 2008). A neurocognitive model was recently suggested to explain the immersive nature of art, particularly films, which proposed that under proper context and mind set, the viewer's central executive hands-over control to the film information, resulting in a temporary rewarding dissociative state. (Dudai, 2008). This model emphasizes the potential of some forms of art to detach their audience from the surrounding world. It is important to note that the information that forms such a holistic and immersive experience does not have to originate solely from the artwork, some information may also be complemented from intrinsic, self-related processes, as will be discussed below.

The distinctiveness of artistic experience was also extensively addresses by Zeki (Zeki, 1998), elaborating on views of various artists and theorists who emphasized the "gist" aspect of art: "art shows us that there are also constant truths concerning forms" (Mondrian). "Artist... give(s) reality a more lasting interpretation" (Matisse). "The whole beauty and grandeur of Art consists ... in being able to get above all singular forms, local customs, particularities of every kind ... makes out an abstract idea of their forms ..." (John constable) (quotes from Zeki, 1998). Along the same lines, Zeki presents the views of Hegel and Kant who deem art as being able to represent reality better than "ephemera of sense data," proposing that the brain, which has representation of concepts, can, when viewing art, easily extract them in a simpler manner than when viewing real-world perceptions. "Art furnished us with the things themselves, but out of the inner life of the mind" (Hegel, quote from Zeki, 1998). This point of view suggests two potential values for training: one concerning art as an abstract and generalized representation, and the other concerning the necessity the art induces to complete the experience from "the inner mind" thus using imagery and internal mentation.

The first aspect, regarding art as abstract and generalized representation, proposes that art extracts and presents the essence of things. Zeki further suggested that art and the brain share this capacity (Zeki, 1998), both seeking to find constancies in the world, and to represent the essence of objects and events given the dynamic and variable world around. This proposed characteristic of art again may imply that art can be a vehicle for improvement of representation, and allow more efficient access and modification of brain representations. This may suggest 
that learning through artistic experience can be more effective and more prone to generalization. Interestingly, this notion of a generalized representation, which is invariant of specific details and exemplars, resembles ideas behind the prevailing neural network models of associative memory (Hertz et al., 1991; Amit, 1995). In these models, memories are represented as stable states of network activity, called attractors, each attractor being the memory representation of all input stimuli that are drawn to it through network dynamics. Attractors are typically formed by Hebbian learning of overlapping patterns of synaptic efficacies (Hebb, 1949; Hertz et al., 1991; Amit, 1995), and thus the learned memory representations are formed by the specific history of experiences (Blumenfeld et al., 2006).

The second aspect, the necessity to complete the experience from "the inner mind," addresses another possible advantagethe self-driven activation of some brain representations as opposed to pure externally driven activation. Indeed, artworks require the experiencer to "complete the experience" using imagination and other internally generated cognitive processes to fill in the gap to create a holistic experience. Imagery is known to be a useful tool for cognitive training in physiotherapy. For example, it was demonstrated that training for stroke patients which included motor imagery in addition to occupational therapy was more effective than training with occupational therapy alone (Page et al., 2001). In addition, filling-in the gap by "inserting" the viewers own subjective self experience, is possibly what allows for enhanced immersion and identification. Self-related thought processes, such introspection, self-representation, autobiographic and prospective memory, and volition, are suggested be mediated by a network of brain regions called the default-mode network (Gusnard and Raichle, 2001; Buckner et al., 2008; Preminger et al., 2010b). Such self-driven processes, combined with the externally driven ones are possibly what make the experience comprehensive. Clearly, different forms of art vary in the gap they leave between the provided and self-generated information. This can be considered and used depending on the goals of the transformative artwork.

Another interesting aspect can be derived from theory of performing arts (Schechner, 2002). It is suggested that the performing art entail an aspect of "re-doing" on top of to the "doing" itself. Re-doing means that any activity is a restored behavior, thus the activity comes in context of previous instances, and these restored behaviors can be "worked on ...played with, made into something else ... [even] transformed" (Schechner, 2002). More broadly, the artist can be thought of as "re-doing" a real-life experience of the experiencer. He drives the experiencer to reexperience some "basic" stored experience, but in a manipulated way. Thus, the artist can choose to emphasize a specific aspect of the experience, decide how emotional the experience will be, to what context it will be put in, etc.

As opposed to scientists, who are specialists in breaking down the cognitive experience into pieces, artists specialize in activation of multiple cognitive functions in cohort, generating holistic experiences involving perception, executive functions, intrinsic processes, and sometimes even action. Whereas researchers of visual perception commonly study simple and isolated stimuli to study the visual cortex (Sagi, 2010), painters, film makers, and video game designers are experts in inducing experiences by complex visual stimuli, thus causing a wide spread brain activation, where different brain networks work in cohort (in cinema, Hasson et al., 2004). Thus, possibly, the experience of art, can be placed somewhere in between scientific paradigms and real-life experience. It is "engineered" and has the essence of the experience but on the other hand it is also holistic and comprehensive.

\section{ART AS LONG-TERM TRANSFORMATIVE EXPERIENCE}

Currently many artworks are seen by observers on a singleexposure basis, in a museum, gallery, or theater, and are created as such. However, some artworks do get repetitive exposure by their audiences, intentionally or un-intentionally. A good example is art which is presented in the public space (e.g., artworks by Christo, Anish Kapoor) where people have the chance to experience it again and again when passing by. Another example is recorded music, where a particular piece of music may be heard again and again. Similarly a painting which is hanged in the corridor of an office building, or in a living room, is experienced repetitively every day. How does the experience change from exposure to exposure? Does it leave any accumulating long-term mark on observers' cognition and brain? Would the artworks be designed differently if the repetitive exposure effect would have been taken into account? For instance, when seeing the same painting in your office corridor day by day, you probably already ignore it. What if it would have been a video-art that slightly changes every day? Will you notice? What effect could it have on you? These questions are for artists and researchers to answer.

Neuroscience methods offer a good starting point for developing systematic measures of the impact of long-term exposure to art on specific cognitive capabilities of the viewer, and to the unique contribution of the artistic aspects to these effects. Specifically, studies of experience-based plasticity utilize various cognitive tasks and neuropsychological assessments (Lezak, 1995) to evaluate changes in various cognitive functions (e.g., Mahncke et al., 2006; Chein and Morrison, 2010; Preminger et al., 2010a). Similar tools can be used to study the effect of longterm repeated artistic experience, as done in (Green and Bavelier, 2006b; Green et al., 2010). Assessing the unique effect of artistic experiences as opposed other experiences is more complex and touches upon philosophical questions regarding the definition of artistic experience, which is out of the scope of this review. Ignoring these philosophical aspects, and assuming commonly accepted views of art, some recent approaches taken in neuroesthetics literature present attempts of comparing artistic experience to non-artistic experience in studies examining brain activation by art. One approach is by comparing artistic stimuli to similar natural stimuli (e.g., Dio et al., 2011, comparing photos of sculptures of human body with photos of real bodies, or comparing paintings to scrambled paintings in Wiesmann and Ishai, 2010). Another approach compares different genres of art (e.g., comparing representational and indeterminate paintings in Ishai et al., 2007). Similar comparisons can be used to study the unique effects of long-term exposure to art, for example comparing people who walk to their work through a park with 
sculptures/art with people walking to their work via the same or a similar part without sculptures, or comparing effects playing different genres of video games or hearing types of music. Such approaches may provide insights to what are the effective components in different art forms and genres. Another approach that can be used is the comparison between artists to non-artists as described above.

Utilizing continuing experience to induce long-term change of neuropsychological functions may lead to a novel viewpoint on art and a different approach to its creation. Artists can design artworks that aspire to form, in addition to a one-shot influence on experience and affect, continuing experiences which accumulate into to a lasting change on specific neurocognitive functions. Similarly to the questions an artist asks today: what meaning or experience do I want to convey to my audience? How will I convey it? Artists could consider the questions: what lasting change do I want to induce by on-going exposure and on which neurocognitive functions? How can I induce it? Principles of experience-based plasticity discovered within the perceptual, motor, emotional, and executive domains, together with the experience and knowledge acquired in the arts, could form the foundation for this new approach to art as a continuing transformative experience.

\section{REFERENCES}

Achtman, R. L., Green, C. S., and Bavelier, D. (2008). Video games as a tool to train visual skills. Restor. Neurol. Neurosci. 26, 435-446.

Amit, D. J. (1995). The Hebbian paradigm reintegrated: local reverberations as internal representations. Behav. Brain Sci. 18, 617-657.

Beardsley, M. C. (1958). Aesthetics. Indianapolis, IN: Hackett.

Bengtsson, S. L., Csíkszentmihályi, M., and Ullén, F. (2007). Cortical regions involved in the generation of musical structures during improvisation in pianists. J. Cogn. Neurosci. 19, 830-842.

Berkowitz, A. L., and Ansari, D. (2008). Generation of novel motor sequences: the neural correlates of musical improvisation. Neuroimage 41, 535-543.

Bezzola, L., Mérillat, S., Gaser, C., and Jäncke, L. (2011). Training-induced neural plasticity in Golf Novices. J. Neurosci. 31, 12444-12448.

Blumenfeld, B., Preminger, S., Sagi, D., and Tsodyks, M. (2006). Dynamics of memory representations in networks with novelty-facilitated synaptic plasticity. Neuron 52, 383-394.

Boot, W. R., Kramer, A. F., Simons, D. J., Fabiani, M., and Gratton, G. (2008). The effects of video game playing on attention, memory, and executive control. Acta Psychol. 129, 387-398.
Buckner, R. L., Andrews-Hanna, J. R. and Schacter, D. L. (2008). The brain's default network: anatomy, function, and relevance to disease. Ann. N.Y. Acad. Sci. 1124, 1-38.

Buonomano, D. V., and Merzenich, M. M. (1998). Cortical plasticity: from synapses to maps. Annu. Rev. Neurosci. 21, 149-186.

Chein, J. M., and Morrison, A. B. (2010). Expanding the mind's workspace: training and transfer effects with a complex working memory span task. Psychon. Bull. Rev. 17, 193-199.

Dio, C. D., Canessa, N., Cappa, S. F., and Rizzolatti, G. (2011). Specificity of aesthetic experience for artworks: an fMRI study. Front. Neurosci. [Special issue on Brain and Art, under review], 5, 1-14.

Dewey, J. (1934). Art as Experience. New York, NY: Penguin Books Ltd.

Dorval, M., and Pepin, M. (1986). Effect of playing a video game on a measure of spatial visualization. Percept. Mot. Skills 62, 159-162.

Drew, D., and Waters, J. (1986). Video games: utilization of a novel strategy to improve perceptual motor skills non-institutionalized elderly. Cogn. Rehabil. 4, 26-31.

Dudai, Y. (2008). Enslaving central executives: toward a brain theory of cinema. Projections 2, 21-42.

Dye, M. W. G., Green, C. S., and Bavelier, D. (2009). Increasing speed of processing with action video and cognitive functioning in the

\section{CONCLUSIONS}

This review links between the research of experience-based plasticity and the on-going and repeated experience of art in its various forms. The integration of knowledge from these different fields offers a potential for both disciplines. Scientists who study plasticity and develop paradigms to induce long-term neurocognitive changes may benefit from the various advantages of the holism, engagement, and the "gist" that art experiences offer. On the other hand, artists may use the experience-based plasticity approach to extend their aesthetic creations from immediate oneshot experiences to on-going or repeated experiences that induce long-lasting neurocognitive effect.

\section{ACKNOWLEDGMENTS}

I would like to thank Barak Blumenfeld, Lior Noy, Oran Singer, and the referees for critical reading of earlier versions of this manuscript, and Tanya Preminger, Tamar Erez, Ehud Ben-Yaakov, Uri Hasson, Eydor Diamant, and Guido Hesselmann for fruitful discussions. This manuscript was partially made possible through the support of a grant from the John Templeton Foundation. The opinions expressed in this publication are those of the author and do not necessarily reflect the views of the John Templeton Foundation.

games. Curr. Dir. Psychol. Sci. 18, 321-326.

Ericsson, K. A., Krampe, R. T., and Tesch-Römer, C. (1993). The role of deliberate practice in the acquisition of expert performance. Psychol. Rev. 100, 363-406

Feng, J., Spence, I., and Pratt, J. (2007). Playing an action videogame reduces gender differences in spatial cognition. Psychol. Sci. 18, 850-855.

Green, C. S., and Bavelier, D. (2003). Action video game modifies visual selective attention. Nature 423, 534-547.

Green, C. S., and Bavelier, D. (2006a). "The cognitive neuroscience of video games," in Digital Media: Transformations in Human Communication, eds P. Messaris and L. Humphreys (New York, NY: Peter Lang), 211-224.

Green, C. S., and Bavelier, D. (2006b). Effects of action video game playing on the spatial distribution of visual selective attention. J. Exp. Psychol. Hum. Percept. Perform. 32, 1465-1478.

Green, C. S., and Bavelier, D. (2008). Exercising your brain: a review of human brain plasticity and training-induced learning. Psychol. Aging 23, 692-701.

Green, C. S., Pouget, A., and Bavelier, D. (2010). Improved probabilistic inference as a general learning mechanism with action video games. Curr. Biol. 20, 1573-1579.
Gusnard, D. A., and Raichle, M. E. (2001). Searching for a baseline: functional imaging and the resting human brain. Nat. Rev. Neurosci. 2, 685.

Hasson, U., Nir, Y., Levy, I., Fuhrmann, G., and Malach, R. (2004). Intersubject synchronization of cortical activity during natural vision. Science 303, 1634-1640.

Hasson, U., Landsman, O., Knappmeyer, B., Vallines, I., Rubin, N., and Heeger, D. J. (2008). Neurocinematics: the neuroscience of making movies. Projections 1, 2-26.

Hasson, U., Malach, R., and Heeger, D. (2009). Reliability of cortical activity during natural stimulation. Trends Cogn. Sci. 14, 40-48.

Hebb, D. O. (1949). The Organization of Behavior. New York, NY: Wiley.

Hertz, J., Krogh, A., and Palmer, R. G. (1991). Introduction to the Theory of Neural Computation. Redwood City, CA: Addison-Wesley.

Hlustík, P., and Mayer, M. (2006). Paretic hand in stroke: from motor cortical plasticity research to rehabilitation. Cogn. Behav. Neurol. 19, 34-40.

Ishai, A. (2011). "Art compositions elicit distributed activation in the human brain," in Aesthetic Science: Connecting Minds, Brains, and Experience, eds A. P. Shimamura and S. E. Palmer (New York, NY: Oxford University Press), 337-355. Ishai, A., Fairhall, S. L., and Pepperell, R. (2007). Perception, memory 
and aesthetics of indeterminate art. Brain Res. Bull. 73, 314-324.

Jancke, L. (2009). The plastic human brain. Restor. Neurol. Neurosci. 27, 521-538.

Jenkins, H. (2000). Art form for the digital age. Technol. Rev. Available online at: http://www.technology review.com/computing/12189/

Kandel, E. R. (2001). The molecular biology of memory storage: a dialogue between genes and synapses. Science 294, 1030-1038.

Kandel, E. R., Schwartz, J., and Jessell, T. (2000). Principles of Neural Science. 4th edn. New York, NY: McGrawHill.

Karni, A., Meyer, G., Rey-Hipolito, C., Jezzard, P., Adams, M. M., Turner, R., and Ungerleider, L. G. (1998). The acquisition of skilled motor performance: fast and slow experience-driven changes in primary motor cortex. Proc. Natl. Acad. Sci. U.S.A. 95, 861-868.

Karni, A., and Sagi, D. (1991). Where practice makes perfect in texture discrimination: evidence for primary visual cortex plasticity. Proc. Natl Acad. Sci. U.S.A. 88, 4966-4970.

Kelly, A. M. C., and Garavan, H. (2005). Human functional neuroimaging of brain changes associated with practice. Cereb. Cortex 15, 1089-1102.

Koch, C. (2004). The Quest for Consciousness: A Neurobiological Approach. Englewood, CO: Roberts \& Company.

Kolb, B., and Whishaw, I. Q. (1998). Brain plasticity and behavior. Annu. Rev. Psychol. 49, 43-64.

Kottlow, M., Praeg, E., Luethy, C., and Jancke, L. (2011). Artists' advance: decreased upper alpha power while drawing in artists compared with non-artists. Brain Topogr. 23, 392-402.

Kourtzi, Z., and DiCarlo, J. J. (2006). Learning and neural plasticity in visual object recognition. Curr. Opin. Neurobiol. 16, 1-7.

Kroll, J. (2000). 'Emotion Engine'? I Don't Think So. Newsweek. March 6.

Lezak, M. D. (1995). Neuropsychological Assessment. Third Edn. USA: Oxford University Press.

Li, S., Mayhew, S. D., and Kourtzi, Z. (2009). Learning shapes the representation of behavioral choice in the human brain. Neuron 62, 441-452.

Limb, C. J., and Braun, A. R. (2008). Neural substrates of spontaneous musical performance: an fMRI study of Jazz improvisation. PLoS One 3:e1679. doi: 10.1371/journal.pone.0001679

Loya, A., Hesselman, G., Malach, R., and Preminger, S. (2010).
"Neural activity associated with self-paced overt word generation an fMRI study," Israeli Society for Neuroscience 19th Annual Meeting, Israel. [Paper in preparation].

Ludmer, R., Dudai, Y., and Rubin, N. (2011). Uncovering camouflage: amygdala activation predicts long-term memory of induced perceptual insight. Neuron 69, 1002-1014.

Mahncke, H. W., Connor, B. B., Appelman, J., Ahsanuddin, O. N., Hardy, J. L., Wood, R. A., Joyce, M. N., Boniske, T., Atkins, S. M., and Merzenich, M. M. (2006). Memory enhancement in healthy older adults using a brain plasticity-based training program: a randomized, controlled study. Proc. Natl. Acad. Sci. U.S.A. 103, 12523-12528.

McClure, S. M., Li, J., Tomlin, D., Cypert, K. S., Montague, L. M., and Montague, P. R. (2004). Neural correlates of behavioral preference for culturally familiar drinks. Neuron 44, 379-387.

Mesulam, M. M. (2002). "The human frontal lobes: transcending the default mode through contingent encoding," in Principles of Frontal Lobe Function, eds D. T. Stuss and R. T. Knight (New York, NY: Oxford University Press), 8-30.

Meyer, M., Elmer, S., Ringli, M., Oechslin, M. S., Baumann, S., and Jancke, L. (2011). Long-term exposure to music enhances the sensitivity of the auditory system in children. Eur. J. Neurosci. 34, 755-765.

Nachmanovitch, S. (1990). Freeplay: Improvisation in Life and Art. New York, NY: Penguin Putnam Inc.

Newell, A., and Rosenbloom, P. S. (1981). "Mechanisms of skill acquisition and the law of practice," in Cognitive Skills and their Acquisition, ed J. R. Anderson (New Jersey, NJ: Lawrence Erlbaum Associates), 1-55.

Page, S. J., Levine, P., Sisto, S., and Johnston, M. V. (2001). A randomized efficacy and feasibility study of imagery in acute stroke. Clin. Rehabil. 15, 233-240.

Pascual-Leone, A., Amedi, A., Fregni, F., and Merabet, L. B. (2005). The plastic human brain cortex. Annu. Rev. Neurosci. 28, 377-401.

Polat, U., Ma-Naim, T., Belkin, M., and Sagi, D. (2004). Improving vision in adult amblyopia by perceptual learning. Proc. Natl. Acad. Sci. U.S.A. 101, 6692-6697.

Preminger, S. (2009a). Improvisation for neurorehabilitation. Front. Neurosci. [Special issue on Augmenting Cognition].
Preminger, S., Blumenfeld, B., Sagi, D., and Tsodyks, M. (2009b). Mapping memories of gradually changing objects. Proc. Natl. Acad. Sci. U.S.A. 106, 5371-5376.

Preminger, S. (2011). "Improvisation for prefrontal rehabilitation," Chapter 2 in Augmenting Cognition, eds H. Markram and I. Segev (Switzerland: EPFL Press), 41-68.

Preminger, S., Wertman, M., Bogen, Y., Heled, E., Itskovich, E., Zigdon, H., Loya, A., Hesselmann, G., Malach, R., and Agranov, E. (2010a). "Verbal fluency as a training method for apathy in TBI patients: methods and preliminary results," Conference of Israeli Rehabilitation Association, Israel. (Paper in preparation).

Preminger, S., Harmelech, T., and Malach, R. (2010b). Stimulus-free thoughts induce differential modulation of the default system in the human brain. Neuroimage 54 1692-1702

Preminger, S., Harmelech, T., Laufer, O., Gilaie-Dotan, S., Arieli, A., and Malach, R. (2008). Functional magnetic resonance imaging study of self-initiated purposeful behavior in the human cerebral cortex. Soc. Neurosci. USA. [Paper in preparation].

Preminger, S., Sagi, D., and Tsodyks, M. (2007). Effects of perceptual history on memory of visual objects. Vision Res. 47, 965-973.

Ramachandran, V. S., and Hirstein, W. (1999). The science of art A neurological theory of aesthetic experience. J. Conscious. Stud. 6, 15-51.

Recanzone, G. H., Schreiner, C. E., and Merzenich, M. M. (1993). Plasticity in the frequency representation of primary auditory cortex following discrimination training in adult owl monkeys. J. Neurosci. 13, 87-103.

Roediger, H. L., Dudai, Y., and Fitzpatrick, S. M., eds. (2007). Science of Memory: Concepts. New York, NY: Oxford University Press.

Rothschild, M. L., Thorson, E., Reeves, B., Hirsch, J., and Goldstein, R. (1986). EEG activity and the processing of television commercials. Commun. Res. 13, 182-220.

Rothschild, M., and Hyun, Y. J. (1990). Predicting memory for components of TV commercials from EEG. $J$. Consum. Res. 16, 472-478.

Sagi, D. (2010). Perceptual learning in vision research. Vision Res. 51, 1552-1566.

Schechner, R. (2002). Performance Studies: An Introduction. London: Routledge/Taylor and Francis.
Schneider, P., Sluming, V., Roberts, N., Scherg, M., Goebel, R., Specht, H. J., Dosch, H. G., Bleeck, S., Stippich, C., and Rupp, A. (2005). Structural and functional asymmetry of lateral Heschl's gyrus reflects pitch perception preference. Nat. Neurosci. 8, 1241-1247.

Schwartz, J. H. (1982). Molecular biology of learning: modulation of transmitter release. Science 218, 433-444.

Smith, M. L., Gosselin, F., and Schyns, P. G. (2006). Perceptual moments of conscious visual experience inferred from oscillatory brain activity. Proc. Natl. Acad. Sci. U.S.A. 103, 5626-5631.

Smuts, A. (2005). Are Video Games Art? Contemp. Aesthetics 3 . Available online at: http://www. contempaesthetics.org/newvolume/ pages/article.php?articleID=299

Stewart, L. (2008). Do musicians have different brains? Clin. Med. 8, 304-308.

Vogt, S., and Magnussen, S. (2007). Expertise in pictorial perception: eye-movement patterns and visual memory in artists and laymen. Perception 36, 91-100.

Wiesmann, W., and Ishai, A. (2010). Training facilitates object recognition in cubist paintings. Front. Hum. Neurosci. 4:11. doi 10.3389/neuro.09.011.2010

Yarbuz, A. L. (1976). Eye-Movement and Vision. Trans. New York, NY: Plenum Press.

Zeki, S. (1998). Art and the brain. Daedalus 127, 71-103.

Zeki, S. (1999). Inner Vision: An Exploration of Art and the Brain. New York, NY: Oxford University Press.

Conflict of Interest Statement: The authors declare that the research was conducted in the absence of any commercial or financial relationships that could be construed as a potential conflict of interest.

Received: 12 April 2011; accepted: 02 April 2012; published online: 24 April 2012.

Citation: Preminger S (2012) Transformative art: art as means for long-term neurocognitive change. Front. Hum. Neurosci. 6:96. doi: 10.3389/fnhum.2012.00096

Copyright (c) 2012 Preminger. This is an open-access article distributed under the terms of the Creative Commons Attribution Non Commercial License, which permits non-commercial use, distribution, and reproduction in other forums, provided the original authors and source are credited. 